\title{
La división del trabajo cognitivo en la prueba pericial: el juez, las partes, los peritos y las comunidades expertas
}

\author{
The Division of Cognitive Labor in Expert \\ Evidence: Judges, Parties, Experts and \\ Their Communities
}

Carmen Vázquez

Resumen: El presente trabajo ofrece respuesta a las réplicas planteadas a mi trabajo "El diseño normativo de las pruebas periciales, a propósito del razonamiento inferencial de los expertos y la comprensión judicial”, realizadas por Florencia Rimoldi y Rachel Herdy. Como el título señala, básicamente refiero aquí a la división del trabajo cognitivo que hay (o debería haber) cuando analizamos las pruebas periciales de manera amplia y que implica no solo a los jueces, sino a las partes, los peritos que participan en el proceso judicial y las comunidades expertas a las que estos pertenecen. Palabras claves: Prueba pericial, práctica de la prueba, deferencia a los expertos.

Abstract: In this paper I answer to some replies raised by Florencia Rimoldi and Rachel Herdy to my paper "The Institutional Design of Expert Evidence. Remarks on the Inferential Reasoning of Experts and Judicial Comprehension". As the title indicates, basically I argue that when we ana-

* Doctora en Derecho y Profesora. Universitat de Girona, Girona, España. Correo electrónico: carmen.vazquez@udg.edu. 
lyze expert evidence as a whole, there is (or should be) a division of cognitive labor among judges, parties, experts and the communities to which they belong.

Keywords: Expert evidence, presentation of evidence at the trial, deference to experts.

En primer lugar, quiero agradecer muy sinceramente a Florencia Rimoldi y Rachel Herdy por sus muy interesantes observaciones críticas a mi trabajo "El diseño normativo de las pruebas periciales, a propósito del razonamiento inferencial de los expertos y la comprensión judicial". ${ }^{1}$ El "principio de contradicción" no solo es fundamental para la justificación y corrección de las decisiones judiciales, también lo es en los mismos términos para las ideas que se defienden en la academia. Así, cuando se someten a contradicción los argumentos es posible evaluar mejor su rendimiento y decidir entonces cuáles de ellos es necesario modificar y cuáles de ellos han sobrevivido a la crítica. Por supuesto, al igual que sucede en el proceso judicial, lo anterior solo es posible cuando quien ejerce la contradicción presenta argumentos relevantes y sólidos.

Pues bien, por lo que parece, la parte de mi trabajo que considero más novedosa y que, en realidad, desde mi perspectiva es el núcleo central de las tesis allí defendidas ha quedado intacta. Mi trabajo pretende ofrecer un conjunto de herramientas procesales que deberían estar en nuestras legislaciones para que los jueces (y las partes) puedan lidiar de mucho mejor manera con las complejidades que las tan variadas pruebas periciales en la actualidad presentan. Desafortunadamente seguimos teniendo legislaciones (y, a veces, criterios jurisprudenciales) no solo ancladas al pasado en cuanto al razonamiento probatorio en general se refiere, y en las que poca atención se ha prestado a las dificultades reales de las pruebas periciales, sino que asumen una concepción de la prueba pericial demasiado simplista y a veces hasta con una imagen romántica de la empresa científica en el fondo.

1 También quiero agradecer enormemente a los editores de Discusiones por pensar en mí para este número; muy especialmente agradezco también a Valeria Trotti, por sus gestiones y todo el trabajo que le ha supuesto este número. 
La división del trabajo cognitivo en la prueba pericial: ...

Junto a lo anterior, también queda intacto otro de los desarrollos que hago en mi trabajo y que, a día de hoy, parece no habitual en la literatura procesal, por ejemplo. Me refiero a la importancia de la práctica de las pruebas a efectos de un tratamiento adecuado para la prueba pericial en particular, para su correcta valoración en los casos concretos en que es empleada. La mayoría de la literatura en nuestro ámbito cultural, e incluso de la jurisprudencia, pone el énfasis en la valoración de la prueba pericial, obviando que aquello que suceda en la práctica durante el juicio oral será fundamental para la atribución de valor probatorio a este elemento de juicio. Actúan como si la atribución de valor probatorio surgiera solo de la mente del juez a partir de haber leído o escuchado lo que dijo el experto.

Mi énfasis en la práctica de la prueba pericial y las herramientas procesales que podemos concebir en esa etapa para mejorar la información disponible tienen como objetivo lograr la comprensión judicial sobre el conocimiento experto relevante para la toma de decisión de los hechos en un caso concreto. Tanto Rimoldi como Herdy se enfocaron en debatir los presupuestos que subyacen a mi propuesta: una con más énfasis en las bases epistemológicas y la otra acentuando cierto supuesto empírico-psicológico respecto de la posibilidad de la comprensión judicial para la valoración de estas pruebas. Ambas sugieren un modelo total o parcialmente distinto de la prueba pericial basado, por lo que parece, en sus críticas correspondientes; aunque, muy llamativamente para mí, sin prestar atención precisamente a la práctica de las pruebas como una etapa procesal determinante.

En lo que sigue, como es evidente, me detendré a intentar responder las observaciones de Rimoldi y Herdy, a veces para aclarar al lector mis argumentos presentados en el trabajo que se discute, otras quizá para matizarlos a partir de sus estupendas observaciones y algunas para contraargumentar sus concepciones. Para ello, dividiré mi respuesta en tres apartados. En el primero, me dedicaré a ahondar en la idea de "modelo de la prueba pericial", poniendo particular atención al posible rol de la deferencia en las distintas propuestas que se discuten trayendo al debate la división del trabajo cognitivo presente en nuestras sociedades del conocimiento actuales. ${ }^{2}$

2 "Division of cognitive labor" es una expresión de Philiph Kitcher, un conocido filósofo de la ciencia, título de uno sus célebres artículos donde discute la división del trabajo cognitivo 
En el segundo apartado, me dedicaré a tratar los "criterios indirectos" que ambas, aunque desde perspectivas muy diversas, sugieren para complementar (en el caso de Rimoldi) o para apartarse (en el caso de Herdy) de mi presupuesto de que los jueces deben comprender el razonamiento pericial. Finalmente, en el tercer apartado, de manera breve haré algunas referencias a la idea de Rimoldi de atender al contextualismo en el marco de una dinámica más argumentativa, a diferencia de ella pondré el acento en el rol de las partes y otras consideraciones jurídicas.

\section{Un modelo de prueba pericial y la deferencia hacia los expertos}

Mi trabajo es claramente normativo, no descriptivo; aunque, por supuesto, tomo en cuenta algunas cuestiones sobre el funcionamiento en la práctica judicial de la prueba pericial. Por ello, en varios momentos hablo del diseño institucional que deberíamos tener y de aquello que debiera ser el objetivo de la valoración judicial de la prueba pericial. Ante ese modelo, el análisis de Herdy plantea un argumento más bien descriptivo: "no hay buenas razones para presuponer que los jueces tienen la disposición o son capaces de comprender las inferencias de los expertos". Más allá de su verdad o falsedad, ${ }^{3}$ la afirmación en sí misma es sumamente llamativa al menos por dos cuestiones:

1. Si tuviéramos que ceñirnos a la disposición o capacidad que de hecho tienen los jueces (o de cualquier otro decisor) para plantearnos cómo debería ser una institución o qué cambios deberíamos imple-

en la ciencia (Kitcher, 1990). La expresión se derivó, a su vez, de la "division of linguistic labor" propuesta en la filosofía del lenguaje por Hillary Putnam, en Putnam (1975). Por supuesto, la expresión es una analogía a la idea de Adam Smith sobre la división del trabajo para el progreso económico mediante cierta especialización de los trabajadores en tareas específicas en la producción. Y, también por supuesto, el título de este artículo sigue en alguna medida esta senda situando al individuo (el juez) dentro del contexto social de otros agentes cognitivos que también participan del proceso judicial en donde tiene que haber cierta distribución óptima de los esfuerzos de cada uno.

3 Lo que es imposible de saber puesto que la afirmación de Herdy no tiene base en ningún estudio empírico sobre esa disposición o capacidad de los jueces. 
La división del trabajo cognitivo en la prueba pericial: ...

mentar, entonces la capacidad de cambio en el sistema sería muy reducida o mínima. En materia de prueba testifical, pensemos: si los jueces no tienen la disposición la capacidad para valorar las declaraciones testimoniales en función de la memoria del sujeto, ¿podríamos sugerir las modificaciones que nos exige hacer la psicología del testimonio en las dinámicas procesales tradicionales en función de lo que ahora sabemos gracias a ella? Según el argumento de Herdy, no, no podríamos; y, entonces, ¿deberíamos seguir conformándonos con que los jueces sigan valorando el lenguaje corporal de los testigos para atribuirle valor a la confiablidad (subjetiva) del testigo?

2. Cuando Herdy se refiere a la "disposición" de los juzgadores, ¿en qué medida está tomando en cuenta a los jueces que señala Devis Echandía, i. e. aquellos que no hacen lo que deben por pereza física y mental? (Devis Echandía, 1972, p. 61). Y, de ser así, ¿por qué deberíamos tomarlos a ellos como referencia para construir un modelo sobre la prueba pericial o cualquier otra institución probatoria o jurídica? Obviamente, deberíamos tomar en cuenta que en todo sistema jurídico podría haber más o menos jueces de ese tipo y pensar, entonces, los incentivos necesarios para evitar que actúen con esa pereza o con otras "disposiciones" no acordes con las exigencias de su función.

Por supuesto, Herdy podría no solo estar contando con el tipo de jueces antes descrito sino estar aludiendo a algo así como el juez promedio. En ese contexto, estaría dejando de lado un elemento importante del contexto en que hace su afirmación sobre la falta de capacidad de los jueces para comprender las inferencias de los expertos: que hasta hace muy poco el interés en analizar el razonamiento probatorio ha sido muy escaso. Las facultades de derecho en nuestros países (de tradición romano-germánica) no han formado a los juristas para pensar la prueba, para identificar las premisas que conformarían el razonamiento fáctico y las inferencias que se justifican a partir de ellas. Esa situación es aplicable a toda la institución probatoria y, por supuesto, hace estragos importantes en la prueba pericial; por ello, no es de extrañar que nuestros jueces ( $\mathrm{y}$ abogados, fiscales, etc.) de hecho tengan serias deficiencias en su razonamiento fáctico. En definitiva, como ha mostrado la teoría racional 
de la prueba, precisamente por ello son aún más importantes modelos que ayuden a los operadores jurídicos a realizar una mejor tarea. ${ }^{4}$

En efecto, pese a sus propios argumentos, Herdy también nos dice que "los jueces tienen el potencial de desarrollar las capacidades necesarias para comprender las inferencias de los expertos", pero se pregunta si entonces bastaría educar a los jueces. Y responde: "me parece [que] no, $\mathrm{o}$ al menos no sin que esto no implique costos muy altos y con resultado todavía inciertos para la administración de la justicia”. Y aquí la verdad es que no es claro qué entiende Herdy por "educarlos"; pero consideremos la literalidad de las palabras y asumamos que se refiere a ciertos cursos en su formación continua sobre distintas áreas del conocimiento experto. Si es así, cobra relevancia una ventaja importante de los jueces profesionales vs. los jurados: a los primeros los podemos formar, tenemos actualmente ya una estructura organizacional y recursos económicos destinados a ello en prácticamente todos nuestros países, mientras que los miembros de los jurados lo son para un solo caso y resultaría imposible darles la formación oportuna. ${ }^{5}$ Los costos serán más o menos altos en función de esa estructura, pero son costos que a mediano plazo podrían ser incluso amortiguados: cada vez los jueces sabrán más y cada vez los jueces mismos podrán formar a otros jueces y establecer jurisprudencia razonable en la materia que ayude al resto de la judicatura. Por supuesto, uno esperaría también que si las facultades de derecho formaran a los estudiantes en razonamiento probatorio tendría un impacto en el tratamiento de la prueba pericial y en la formación de base de los futuros jueces. No se trata, entonces, como Herdy me imputa, de que sea más "plausible presumir que los jueces son más capaces en términos cognitivos que los jurados legos", sino de las

4 Sobre la teoría racional de la prueba puede verse Vázquez (2015, pp. 58-70) y las referencias ahí citadas.

5 Es cierto, como dice Herdy citando a Hans y Saks, que los jurados podrían tener el aspecto favorable de estar conformados por backgrounds diferenciados. Sin embargo, ese argumento (a diferencia de la capacidad para educarlos) es totalmente contingente: depende de cómo de hecho esté conformado cada uno de los jurados. Y, por supuesto, la mera diferencia de background entre ellos no supone que alguno de ellos tenga un background de carácter científico o técnico y mucho menos que sea ese el background relevante para el caso. 
La división del trabajo cognitivo en la prueba pericial: ...

potencialidades que a efectos de formación ofrece cada sistema y, con ello, los costos de tal objetivo. ${ }^{6}$

Sobre la capacidad cognitiva de los jurados, por cierto, vale la pena hacer una aclaración más sobre lo que sabemos al respecto a efectos de decidir el tipo de modelo jurídico procesal: uno de confianza o de desconfianza hacia el decisor. Lo que sabemos, sin embargo, no puede limitarse a la experiencia de cada uno, debe haber estudios empíricos de por medio para conocer más sobre dicha capacidad. Todo estudio empírico, por supuesto, supone una base teórica sobre la parte de la realidad que va a medir y aquí viene una primera cuestión que, en mi opinión, esos estudios deben explicitar: qué capacidades cognitivas podrían considerarse relevantes a efectos de analizar si los actuales decisores son o no cognitivamente competentes para valorar las pruebas periciales. ${ }^{7}$ Algunos estudios que se han llevado a cabo en los Estados Unidos en realidad nos muestran solo ciertas tendencias o incapacidades particulares de algunos jurados sobre la prueba pericial, a partir de los cuales se podría (o no) inferir algo más general sobre su capacidad cognitiva. ${ }^{8}$

Como bien dice Herdy, el artículo de Schauer y Spellman, por ejemplo, muestra cómo los jurados tienden a sobrevalorar las informaciones ofrecidas por expertos y cómo también lo hacen con los testigos. Para cualquiera que lea el artículo en cuestión, es claro que "el argumento de los autores no es que los jurados sean cognitivamente capaces para valorar las pruebas periciales"; yo jamás dije que lo fuera, como me lo imputa Herdy, y no diría

6 Por supuesto, estoy pensando aquí en el tradicional jurado lego que participa una sola vez en su vida como juzgador de los hechos. Es posible, sin embargo, pensar en otras figuras existentes de decisores legos en el derecho que sí gocen de esa potencialidad. Por ejemplo, los llamados "lay magistrates" en North Ireland, que son nombrados por cinco años para decidir, por ejemplo, casos de justicia juvenil junto con un magistrado especialista en derecho. Al respecto, véase: https://judiciaryni.uk/lay-magistrates

7 Herdy sigue a Saks y Spellman (aunque tampoco explica por qué) cuando dice que "teniendo en cuenta que el concepto de capacidad cognitiva - lo cual es clave en el trabajo de Vázquez- hace referencia a la memoria de trabajo que dispone un individuo para el procesamiento consciente de información". Sin embargo, nunca explicita que sería la "memoria de trabajo" y en qué sentido es relevante para mi investigación.

8 También valdría la pena analizar el tipo de casos que serán o fueron parte del estudio, sobre todo la complejidad de las pruebas periciales presentadas y los desacuerdos entre los expertos.

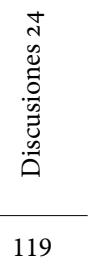


que de mis palabras se pueda inferir semejante cuestión. ${ }^{9}$ Ahora bien, lo que efectivamente no hago, como dice ella, es dedicar mucha atención a los estudios empíricos que se han llevado a cabo en EUA sobre la prueba pericial y la capacidad de los jurados. Y ello se debe a dos razones: 1) el resultado final, como también Herdy reconoce, es que hay estudios empíricos que muestran que los jurados estadounidenses son capaces (en atención a aquello que quieren medir quienes realizan el estudio) y hay otros estudios que muestran que son incapaces (otra vez, en atención a aquello que buscan medir quienes realizan el estudio), por lo que la cuestión no es nada clara, ni siquiera en qué exactamente están midiendo; y 2) el tipo de datos empíricos que ofrecen los estudios mencionados son, como no puede ser de otro modo, contextuales: se mide a determinados sujetos (los miembros de los jurados estadounidenses) en determinadas condiciones (por ejemplo, decidiendo casos más o menos complejos desde el punto de vista de la información experta involucrada). ${ }^{10}$ En definitiva, dadas las enormes diferencias entre los sistemas (y las sociedades en las que rigen) y las tan diversas características de los casos en que están presentes las pruebas periciales (e incluso los tan distintos tipos de pruebas periciales), si no se analiza con cuidado, quizá encontremos datos empíricos para cualquier cosa que queramos sostener sobre la capacidad de los juzgadores de los hechos.

Así, para llevar al terreno de la deferencia la prueba pericial y citando uno de los resultados empíricos descritos por Schauer y Spellman, Herdy pone el énfasis en que "los jurados frecuentemente aciertan, cuando aciertan, porque siguen señales o indicadores periféricos de autoridad epistémica". Como bien dice Herdy, hace tiempo que en el contexto estadounidense Allen y Miller plantearon la dicotomía "deferencia o educación” en

9 Mis palabras textuales son: "Un listado sumamente completo e interesante de los distintos tipos de estudios realizados en Estados Unidos". Por cierto, eso de ninguna manera supone que yo lo tomo como referencia para mi trabajo, como dice Herdy; lo único que hago es remitir al lector a ese trabajo por si le interesa revisar algunos de los estudios empíricos en comento.

${ }^{10}$ Parece que no sería lo mismo medir la capacidad de un jurado para lidiar con información experta compleja en un caso complejo, que en un caso sencillo o con información experta menos compleja.

Un trabajo sumamente interesante, dados los detalles brindados para contextualizar adecuadamente el estudio empírico que realizan sobre las capacidades de los jurados con la prueba pericial en Australia, es Freckelton et al. (2016). 
La división del trabajo cognitivo en la prueba pericial: ...

el tema de la prueba pericial; su idea surgió en un momento en que para la admisión de la prueba pericial se estaba utilizando el criterio Frye, "aceptación general de la comunidad experta", que planteado así es ampliamente deferencialista hacia dicha(s) comunidad(es). ${ }^{11}$ Sin embargo, mi trabajo no pretende realizar un análisis exegético de lo que Allen y Miller querían decir o de lo que Allen busca cuando habla de un modelo educativo; y ello no porque no me parezca interesante ahondar en sus argumentos, sino porque el sistema jurídico del que ellos se encargan, el estadounidense, centra la atención en la admisión de la prueba pericial y, en cambio, deja la cross-examination en manos exclusivamente del adversarialismo feroz entre las partes. En mi modelo, en cambio, me interesa centrarme en la práctica y poner atención no solo en las partes sino también en el juez. Así que más allá de la idea de Allen y Miller, habría que ver si la dicotomía entre deferencia y educación se sostiene y en qué medida.

Aunque Herdy cita mi libro De la prueba científica a la prueba pericial, puede haberse escapado a su atención que ahí desarrollo distintos modelos sobre este elemento de juicio en atención a si se trata de un perito de parte o un perito nombrado judicialmente, siendo el primero más factible para un modelo educativo y el segundo más sensible a un modelo deferencialista. Con lo cual, me parece que la acusación de que "la autora presupone que la deferencia siempre es irracional” en general no me es aplicable y más bien Herdy debería explicitar de manera clara qué entiende por deferencia ${ }^{12}$ y cuándo considera que es racional deferir en otro. En función de sus respuestas podría estar de acuerdo con ella o no.

${ }^{11}$ En esa interpretación literal que se le ha dado por muchos tribunales estadounidenses y la práctica en general. Sin embargo, en el caso Frye el tribunal respectivo completaba ese criterio de la siguiente manera: "aquello de lo que estas pruebas se deducen debe estar lo suficientemente fundado para tener la aceptación general en el área relevante". Al respecto, véase Vázquez (2015, pp. 92-96).

${ }^{12}$ Lo que no es una tarea fácil y al menos habría que distinguir entre la deferencia y acordar con lo que otro dice. Precisamente por ello, me parece problemático un ejemplo de Rimoldi, pues diría que ella está refiriendo precisamente a acordar con los argumentos del otro: "parece razonable deferir ante el/la autor/a en algunas cuestiones, y juzgar por nosotros/as mismos/as la calidad de sus argumentos. Si, luego de haber leído críticamente el artículo, acompañamos la conclusión del/a autor/a, creo que esta actitud es perfectamente razonable, y lo seguiría siendo a pesar de que luego la conclusión resultara falsa”. 
El modelo deferencial crítico que Herdy propone parece basarse de manera exclusiva en la fórmula de Hardwig:

Si $A$ [el lego, el juez] tiene buenas razones para creer que $B$ [el experto] tiene buenas razones para creer que $p$ [la información probatoria], entonces $A$ tiene buenas razones para creer que $p$.

Pero, según Herdy, ¿cuáles son esas buenas razones para creer en B en el contexto procesal? Y ¿qué consecuencia tendría para el diseño normativo aceptar su modelo ${ }^{13}{ }_{i}$ Es compatible deferir en el experto una decisión y el deber del juez de decidir el caso, por más "crítico" que sea?, ¿cómo es compatible ser crítico y deferencialista? Son todas preguntas sin respuesta. ${ }^{14}$ Pero, aún más, en mi opinión, Herdy comete dos errores en su tratamiento: i) poner el énfasis en el argumento de autoridad y obviar las falacias de autoridad, pese a que afirma que "lamentablemente somos bastante buenos en aceptar una conclusión afirmada por alguien que creemos que es una autoridad en un tema"; y, ii) no distinguir la autoridad teórica (sobre qué creer) de la autoridad práctica (sobre qué hacer).

El conocido como "argumento de autoridad" tiene una muy compleja historia en su tratamiento lógico, pues mientras algunos lo han considerado intrínsecamente un tipo de falacia, para otros se trata de un argumento

Por otro lado, Herdy relaciona la deferencia con la aceptación y sugiere la exclusión de las creencias en ese binomio, sin tomar en cuenta que puede haber razones epistémicas para aceptar. El propio Ferrer, a quien ella misma cita y en la misma obra citada (p. 95), dice que en contextos de libre valoración de la prueba “... la creencia del juez en que está probado que p opera como razón (epistémica) para aceptar p. La aceptación superviene a la creencia de que "Está probado que p"'.

${ }^{13}$ Por ejemplo, en mi modelo de la prueba pericial de confianza de los jueces, a efectos de reconstruir cuándo un experto sería confiable, me remito también a Hardwig como una manera para identificar la autoridad teórica de aquel. Sin embargo, dado que mi objetivo es reconstruir la confiabilidad y esta tiene también un aspecto motivacional, más allá de la autoridad teórica, termino decantándome por Hardin. Toda esta reconstrucción, en mi opinión, es adecuada para seleccionar al experto, lo que puede ser una cuestión importante, pero de ninguna manera definitiva o determinante.

${ }^{14}$ Herdy desarrolla mínimamente algunas cuestiones que podrían responder en algunos casos a la primera cuestión, i. e. las buenas razones para creer en B. Su respuesta va en la línea de los criterios indirectos que abordaré en el siguiente epígrafe. 
La división del trabajo cognitivo en la prueba pericial: ...

razonable, genuino y/o legítimo en algunos casos. ${ }^{15}$ Una vez aceptado que el argumento de autoridad es una forma razonable de conocer (lo que en nuestras sociedades actuales altamente especializadas y con cada vez más instituciones evaluando en todos los niveles cierto conocimiento, parecería también ampliamente aceptado), es indispensable analizar cuándo, en cambio, se trata de una falacia de autoridad. La gran mayoría de la literatura relevante considera que el argumento es falaz cuando la supuesta autoridad no lo es en realidad, cuando el área de conocimiento en sí misma es cuestionable o cuando las credenciales del sujeto son de alguna manera pobres (Woods y Walton, 1974, p. 136). Salmon (1984, pp. 98 y ss.), por ejemplo, identificando con mayor precisión tales cuestiones habla de al menos cinco formas en que puede haber un uso indebido del argumento de autoridad: cuando la autoridad es mal interpretada o citada incorrectamente; cuando la supuesta autoridad solo tiene glamur, prestigio o popularidad; cuando los expertos hacen juicios sobre alguna cuestión que sale de su competencia; cuando los expertos hablan de cuestiones sobre las cuales no es posible tener pruebas; y cuando se pone demasiada confianza en los expertos en algunos casos donde hay desacuerdo. ${ }^{16}$

Lo anterior nos aconsejaría andar con sumo cuidado al respecto en el contexto jurídico-procesal, dado que estamos ante un tipo de falacia que podría ser utilizada por los abogados, a veces acostumbrados a argumentar solo con base en el prestigio o la popularidad de sus expertos. Pero también los jueces pueden cometer, por ejemplo, la primera forma de falacia de autoridad haciendo una mala interpretación de las afirmaciones del experto $^{17}$, construyendo así un argumento con una premisa falsa. Precisamente por ello es importante no solo que el juez explicite en la motivación de su decisión qué parte de la prueba pericial está usando, sino también cómo lo está interpretando para el caso concreto; y, para ello, cierto nivel de comprensión es indispensable.

15 Para un interesante análisis histórico sobre los cambios en este tipo de argumento, véase Walton (1997, pp. 32 y ss).

${ }^{16}$ Más recientemente Walton (2008, p. 211) habla de que una apelación a la autoridad es errónea cuando esta es malinterpretada, tomada demasiado en serio o de forma acrítica.

17 También aluden a esta cuestión Woods y Walton (1974, pp. 141-142). 
Por otro lado, Herdy dice que el argumento de autoridad está en la naturaleza de las razones que justifican las decisiones judiciales y traza diversas analogías con el seguimiento de reglas o de la jurisprudencia para ejemplificarlo. Sin embargo, pasa por alto que hay grandes diferencias entre la atribución de autoridad para creer y la atribución de autoridad para actuar de cierta manera. El derecho y las decisiones judiciales que lo aplican pueden ser reconstruidos como una autoridad práctica que nos dice qué debemos hacer, pero no necesariamente hay una pretensión de que los ciudadanos o los jueces crean en lo que deben hacer. Así, por poner un ejemplo sencillo, el derecho puede imponernos la obligación de no fumar, pero no puede obligarnos a creer que no es bueno fumar, de modo que incluso si la reconocemos como autoridad práctica para lo primero, ello no lo hace autoridad teórica para lo segundo. Por ello, creo que Herdy se equivoca cuando dice que "[l]a deferencia a los expertos no difiere del tipo de deferencia que ocurre cuando los jueces deciden aplicar una regla o precedente contrario a sus creencias morales o políticas".

Más allá de las observaciones de Herdy, lo que está en juego cuando se entra al debate del argumento de autoridad y la autoridad teórica es la autonomía sobre el razonamiento. Al respecto, se pronuncia también Rimoldi mencionando algo en lo que estoy parcialmente de acuerdo: "se llega a un punto en la argumentación en juego en el que al juez no le queda más que la deferencia y ello podría ser racional". Yo solo matizaría diciendo que, efectivamente, se podría llegar a ese punto en el proceso dialógico que se da entre los jueces, las partes y los expertos en un proceso judicial. Desde el punto de vista de la epistemología del testimonio, siguiendo a Rimoldi, "habrá algunas premisas o presupuestos del razonamiento cuya verdad la obtiene [el juez] testimonialmente de la misma persona que le está brindado el argumento". Por supuesto, la epistemología social ha mostrado que al menos actualmente es imposible concebir un sujeto cognoscente plenamente autónomo, cuyas creencias estén sustentadas en la experiencia directa o en el razonamiento personal, confiando solo en sus propias facultades cognitivas. No es este el espacio para entrar en las amplias discusiones de la epistemología social, ${ }^{18}$ sin embargo en nuestras sociedades actuales la 
La división del trabajo cognitivo en la prueba pericial: ...

regla es más bien la división del trabajo cognitivo entre los diversos sujetos que la conforman, en la que unos conocen de segunda mano lo que otros conocen en primera persona, y esa dinámica se da tanto entre los propios expertos como entre los legos respecto los expertos.

Quizá una de las características más sobresalientes de la epistemología más clásica es que ha enfocado su atención a un sujeto cognoscente autónomo, independiente de otros agentes cognitivos; ese approach puede ser problemático cuando estamos en un contexto en el que las interacciones entre los diversos sujetos que en él participan son fundamentales para lograr un objetivo epistémico, como es el caso del proceso judicial. En mi trabajo, "El diseño normativo de las pruebas periciales", pude haber enfatizado la autonomía del juez como agente cognoscente, pero ello se debe a que el objetivo que en su momento me plantee era exclusivamente la comprensión judicial. Sin embargo, creo que hay que situar al individuo (el juez) dentro de un contexto social en el que hay otros agentes cognitivos que también tienen un rol que jugar (muchas veces señalado incluso por el propio derecho) no solo en la aportación de información, sino también en el cuestionamiento de la aportada por el otro, y pensar en la distribución óptima de los esfuerzos de cada uno.

Hay que traer al debate, entonces, a las partes del proceso judicial y a las comunidades expertas a las que los peritos pertenecen. Cuando nos centramos solo en la valoración judicial de la prueba pericial sin prestar atención a su conformación y práctica, corremos el riesgo de poner demasiada atención en los jueces y obviar el rol que, para bien o para mal, los otros actores del proceso deben jugar y/o de hecho juegan. Para bien, por ejemplo, porque las partes en ejercicio del contradictorio pueden poner sobre la mesa información sumamente relevante sobre las deficiencias de las afirmaciones del experto; porque las comunidades expertas podrían (y deberían) hacer el tipo de estudios empíricos necesarios para conocer la validez y fiabilidad de los métodos que usan los expertos en lo individual; etc. Para mal, por ejemplo, porque los expertos (por más buenas razones que tengan para creer $\mathrm{X}$ ) también sufren de sesgos cognitivos que los podrían llevar con relativa facilidad a juicios erróneos; ${ }^{19}$ porque las partes

${ }^{19}$ Herdy alude a Kahneman para traer a colación las heurísticas (para poner sobre la mesa la deferencia a los expertos), pero no alude en ningún momento a los sesgos cognitivos a 
a veces no presentan a genuinos expertos o se valen de determinadas credenciales para extender la tarea pericial mucho más allá de la expertise del sujeto, etc. Considerar toda esta compleja dinámica nos lleva a concluir que cuándo "al juez no le queda más que la deferencia” dependerá no solo del caso concreto sino de las interacciones entre los sujetos participantes en él. ${ }^{20} \mathrm{Y}$ para ahondar más ello, pasemos al siguiente apartado analizando el rol de ciertos criterios para valorar la calidad de la prueba pericial a efectos de coadyuvar a la comprensión judicial.

\section{Los criterios indirectos y su uso para distintas deci- siones sobre las pruebas periciales}

Como argumento en el trabajo que se discute, la comprensión judicial es indispensable para la tarea de valorar la prueba pericial, pero ¿qué supone la compresión? Rimoldi da buena cuenta de ella:

La comprensión [...] es un estado epistémico mucho más sofisticado que el mero conocimiento proposicional, y para el caso que nos compete, que es el razonamiento inferencial pericial, queda claro que comprender un razonamiento incluye una capacidad que se encuentra por encima y por debajo de la mera constatación de las premisas, y de la pretensión de que de las premisas se siga una cierta conclusión relevante para el caso.

Un punto sumamente importante que vale la pena resaltar es que la comprensión es gradual, no una cuestión de todo o nada; y, en ese sentido, la división del trabajo cognitivo antes comentada puede ser también una

los que aquellas pueden dar lugar. Se ha desarrollado mucha literatura desde la psicología cognitiva al respecto, y en particular también se han hecho varios experimentos para mostrar la presencia de ciertos tipos de sesgos cognitivos en los expertos. Puede verse, por ejemplo, Dror (2018, p. 243).

${ }^{20} \mathrm{E}$ incluso algunas cuestiones extraprocesales respecto de las comunidades expertas, como se verá en el último apartado sobre el contextualismo en la prueba en el derecho. 
La división del trabajo cognitivo en la prueba pericial: ...

cuestión gradual y, además, es dinámica, porque la comprensión tanto de los expertos como de los legos cambia a lo largo del tiempo. ${ }^{21}$

En el esquema anterior, entonces, la pregunta clave es ¿cómo podemos hacer para que el juez (lego) comprenda las pruebas periciales (expertas) que tiene que valorar? Es una obviedad que el juez no es experto ni pretendemos que lo sea, por lo que su nivel de comprensión será necesariamente inferior en cuanto a la expertise se refiere, aunque no necesariamente lo será en cuanto a los hechos del caso concreto. Y en ese contexto tan específico, un caso concreto, es donde hay que repensar la relevancia de lo que Rimoldi y Herdy identifican como "criterios indirectos" para valorar la prueba pericial y cómo estos ayudarían (o no) en tal labor.

La distinción entre "criterios directos" y "criterios indirectos" para valorar la prueba pericial, en mi opinión, no es tajante; al modo pragmatista uno podría considerarla como una suerte de continuo. Por ejemplo, para conocer la validez y/o la fiabilidad de un instrumento, un criterio directo sería la realización de un estudio empírico controlado que mostrara la validez y/o la fiabilidad del mismo en ciertas condiciones; por otro lado, un criterio indirecto, para valorar lo mismo, sería evaluar la confiabilidad del experto $\mathrm{E}$ o grupo de expertos $\mathrm{G}$ que lo diseñaron. Es indirecto porque a partir de la confiabilidad de E infiero la fiabilidad del instrumento; pero ¿las inferencias no están también presentes en los criterios directos? ¡Por supuesto! A partir de muy diversa información recabada en el estudio se infiere determinada conclusión, no es que se trate de una mera observación simple y sencilla. ${ }^{22}$ Por ende, una cuestión clave parece radicar en la

${ }^{21}$ Sigo aquí a Haack (2020) cuando introduce ciertos matices a la división del trabajo lingüístico de Putnam.

22 Esto se parece mucho a la distinción (artificial) entre "indicio" y "prueba" que impregna al ámbito probatorio. Las pruebas circunstanciales o los indicios son precisamente el tecnicismo con el que se conoce a los hechos a partir de los cuales se infiere(n) otro(s). También se les conoce como "pruebas indirectas", porque no prueban directamente el supuesto de hecho previsto normativamente, sino que llegar a él supone una serie de inferencias. Es esa cadena de inferencias necesaria para llegar al supuesto de hecho previsto normativamente la que ha llevado a la doctrina a considerar que los indicios son más débiles que las pruebas directas, puesto que éstas últimas no requerirían supuestamente de una actividad inferencial. Por ello, se dice, los indicios solo serían aplicables en ausencia de pruebas, negándose incluso el carácter de "prueba" a aquellos. Sin embargo, si prueba quiere decir 
fuerza de la inferencia que conecta al criterio usado con lo que queremos saber, i. e., en la solidez del argumento inductivo en el que descansarían ciertos criterios.

La jurisprudencia y literatura procesal sobre la prueba pericial están repletas de criterios que buscan ayudar a los jueces a valorarla y/o que de hecho ellos emplean para tal objetivo. Uno podría clasificar los diversos criterios atendiendo al objeto que estarían evaluando: criterios dependientes del background de juez (la sana crítica, el libre convencimiento o el prudente arbitrio del juzgador); criterios dependientes del experto individual (credibilidad personal del perito o su credibilidad en el plano científico, sus credenciales o cualificaciones profesionales, su claridad expositiva, la ausencia de contradicciones en su informe); criterios dependientes de ciertas formas procesales (su "institucionalización" jurídica, $v$. gr., estar en la lista oficial de peritos) o incluso criterios netamente numéricos, como la conformidad entre dos o más peritos. Ante este tipo de listados, sistemáticamente habría que preguntarse al menos dos cuestiones: i. ¿Son todos esos criterios relevantes en todos los casos?; y, muy relacionada con la anterior, ii. ¿En qué etapa procesal lo serían?

La relevancia, como bien sabemos, es una cuestión relacional: $\mathrm{X}$ no es relevante o irrelevante en sí mismo, sino dependiendo de para qué. Ello obliga, en realidad, a tratar la relevancia y la etapa procesal de manera conjunta y empezar a desarrollar una respuesta (siempre incómoda y, a veces, decepcionante) del tipo: los criterios en juego serán relevantes dependiendo del caso y de la etapa procesal en juego. Así, por ejemplo, ¿son relevantes las credenciales de los expertos? ¡Por supuesto! De hecho, el derecho propiamente establece que para que un tercero pueda fungir como perito en el proceso judicial se tienen que mostrar las credenciales que permitan tenerlo como tal, siendo entonces condición necesaria para la admisibilidad de una prueba pericial. ¿Son relevantes las credenciales para considerar que un perito está mejor posicionado que el experto de la contraparte? Por ejemplo, en un caso de mala praxis médica por una operación en la rodiverdad o falsedad de la hipótesis, la distinción entre "indicios" y "prueba" es más bien de grado y no categórica. 
La división del trabajo cognitivo en la prueba pericial: ...

lla, pareciera que está mejor posicionado un traumatólogo que un médico familiar. Sin embargo, que un experto está mejor posicionado que el otro cobraría suma relevancia en la práctica de la prueba porque ahí se pueden hacer preguntas que permitan comparar distintos puntos de vista; podría resultar que la especialización hiciera que los expertos se centraran únicamente en la parte del conocimiento que dominan, los huesos en el caso del traumatólogo, y descuidaran una visión más amplia del caso (la incidencia de las articulaciones o incluso cuestiones funcionales como la pisada en los problemas de rodilla). Por otro lado, ¿es la claridad expositiva relevante? Por supuesto, lo es para todos, pero se enfatiza cuando el oyente es un lego que tiene que leer o escuchar una opinión experta; siempre será más fácil seguir el argumento de quien te habla de manera clara que quien "da vueltas" o enreda los argumentos que tiene. ¿Es esa claridad importante para atribuir valor probatorio? En un sentido muy mínimo, lo favorece, pero lo dicho por alguien que es claro podría de todas maneras no merecer valor probatorio porque lo que afirma es falso. Y así, podría seguir con cada uno de esos criterios antes citados; pero, vale la pena volver a los criterios indirectos sugeridos por Rimoldi y Herdy.

"Los criterios de honestidad y responsabilidad epistémica deberían estar vigentes en todas las etapas del proceso", nos dice Rimoldi. Y sigue, "[1]a importancia de que un/a perito/a sea responsable en sus dichos y en la estimación de los márgenes de error es casi tan alta como la confiabilidad de los métodos utilizados". Ahora bien, nos dice, "en la etapa de admisión, la responsabilidad epistémica de un perito [...] podría rastrearse con datos como si la persona tiene suficientes publicaciones sujetas a revisión de pares". Siguiendo la idea antes mencionada sobre la solidez del argumento inductivo, vamos paso a paso:

1. Asumimos que la estimación de los márgenes de error es relevante para atribuir valor probatorio a la prueba $Z$. Esa estimación la puedo conocer, como tercero (sea experto o sea lego), a partir de lo que el experto dice en juicio y/o de sus publicaciones.

2. Sus publicaciones me dicen cuál es su estimación de los márgenes de error. 
3. Su responsabilidad epistémica me dice algo sobre sus dichos respecto de su estimación de los márgenes de error.

El tercer paso, por supuesto, supone mayores saltos inferenciales; pero el segundo paso todavía está incompleto. Herdy que, a su vez, cita a Haack, nos dice:

Por ejemplo, con relación al indicador "publicación en revista científica revisada por pares", uno de los factores de fiabilidad de la prueba pericial estipulados en el caso Daubert, Haack sugiere las siguientes preguntas: Cuando el artículo fue revisado por pares, ¿se le pidió al autor que él mismo indicara los árbitros? ¿Cuántos árbitros fueron consultados? ¿Cuál es el status o la clasificación de la revista? ¿Cómo se sustenta financieramente la revista? ¿Ha habido alguna forma de retractación o expresión de preocupación por parte del editor? ¿El trabajo ya recibió muchas citas? Es obvio que tales preguntas apuntan a señales que pueden eventualmente fallar. Lo que importa es que efectivamente exista una correlación entre los indicadores y la fiabilidad de la prueba pericial, y que los juzgadores legos - jurados o jueces - no queden cognitivamente perdidos para ponerlos en funcionamiento. "La idea, si se prefiere decir así, es proporcionar los medios para gradualmente dar un pequeño empujón a los actores jurídicos en dirección a la identificación de mejores expertos y el escrutinio mejor informado del testimonio del experto".

No es baladí que el indicador tratado por Herdy sea uno establecido jurisprudencialmente en el sistema estadounidense para admitir una prueba pericial. También podría servir, como sostiene Rimoldi, como criterio para identificar quién es un experto en la materia en función: a veces lo hacemos a partir de titulaciones formales, a veces de los años de experiencia, a veces por qué actividades ha realizado, qué publicaciones ha hecho y un largo etcétera que depende del ordenamiento jurídico, de la jurisprudencia sobre la materia y, por supuesto, de lo que aleguen las partes. Pero es muy distinto usarlo para elegir a un experto que usarlo para valorar 
La división del trabajo cognitivo en la prueba pericial: ...

la calidad de lo que ese experto dice. Es más, algunas de las preguntas formuladas por Haack me ayudarían a conocer si el experto tiene un conflicto de interés que pueda mermar su independencia y, a su vez, algunos sistemas jurídicos regulan la independencia pericial como condición necesaria para atribuir valor probatorio a un peritaje. Otras preguntas ayudarían, en cambio, a cuestiones más sustantivas sobre el contenido de la prueba pericial, por ejemplo, la retractación o expresión de preocupación del editor sobre el contenido de lo publicado podría disminuir o directamente eliminar cualquier valor de la estimación de los márgenes de error publicada en ese artículo. Entonces, no sería la publicación en sí misma lo importante, sino lo que dice la retractación de la misma sobre el contenido de lo publicado; tampoco sería la responsabilidad epistémica del sujeto, sino la reacción de otros expertos ante la publicación. ${ }^{23}$ Obviamente todo lo anterior supone cierta comprensión del juez sobre cómo funciona la publicación por pares en la ciencia y qué información le puede brindar todo ello. Así, por ejemplo, sobre un método usado por un perito, el juez no puede evaluar dicha calidad porque no es experto, pero sí puede comprender cómo distintas piezas de información le ayudarán a conocer dicha calidad. ${ }^{24}$

Sobre la fiabilidad de los métodos utilizados por los peritos, sobre todo en el ámbito de las ciencias forenses, hay toda una vasta experiencia en el mundo anglosajón que nos muestra las terribles consecuencias del uso de métodos cuya validez y/o fiabilidad desconocen incluso los propios expertos: la identificación de una persona a través del cabello, por

${ }^{23}$ De hecho, me cuesta ver el rol que tienen las virtudes epistémicas, como la responsabilidad. Pensemos en el caso de un odontólogo forense que en sus dichos periciales afirma que nuestra dentadura es única. Hay enormes dudas sobre ese presupuesto en el área y estudios poblacionales que muestran empíricamente su falsedad. Supongamos que, por ello, afirmamos que el odontólogo es irresponsable epistémicamente porque desconoce información de su área que debería tener en cuenta. A efectos de la administración de justicia, ¿es relevante esa irresponsabilidad epistémica para tomar una decisión sobre la prueba pericial o es que lo relevante es la información existente para no atribuirle valor probatorio o al menos para cuestionar al odontólogo en turno? Me parece que claramente lo segundo y no lo primero; y, por ello, no entiendo la contundente afirmación de Rimoldi sobre que la importancia de la responsabilidad epistémica de los dichos y los márgenes de error es tan alta como la confiabilidad de los métodos usados.

${ }^{24}$ Por cierto, también los expertos funcionan así al menos algunas veces. 
ejemplo, o a través de la marca de sus zapatos en la superficie donde pisó o de la marca de su mordida en un objeto. Hay varios grupos de expertos e instituciones que han trabajado y siguen trabajando en ello; y ciertos ordenamientos jurídicos incorporan como criterio de admisibilidad "la fiabilidad del método" o conocer su rango de error. Sin embargo, cuando de la prueba de $\mathrm{ADN}$ se trata, sucede que ya ni los jueces ni los abogados cuestionan si estamos ante un método de identificación válido y fiable, ¿por qué? Porque hay pruebas abrumadoras de que de hecho lo es. ${ }^{25} \mathrm{Y}$ esas pruebas, que son independientes de los hechos de un caso concreto, y más allá de los estudios controlados a los que fueron sometidas en su día, son resultado de una comunidad experta sólida que sigue trabajando no solo en nuevas técnicas para afrontar problemas como la identificación de un perfil cuando hay mezclas de ADN en una muestra, sino incluso en la detección y disminución de sus errores ( $v$. gr. durante el estudio de una mezcla en el laboratorio). Ahora bien, ¿un abogado podría cuestionar la validez y/o la fiabilidad genérica del método empleado para analizar el ADN? ¡Por supuesto! Jurídicamente no suele haber impedimento alguno al respecto en nuestros ordenamientos. Otra cosa es el éxito que ese cuestionamiento tenga: si la muestra empleada para el análisis respectivo es indubitada, se trata de ADN nuclear y es de buena calidad, quizá la respuesta del juez sería que dicha validez y fiabilidad son un hecho notorio. ${ }^{26}$ ¿Los criterios empleados para tratar la prueba de ADN son aplicables a otras pruebas periciales? No: no hay criterios que sirvan para valorar todo

25 Aunque comúnmente hablemos de "la prueba de ADN", hay desde luego distintos métodos para llevar a cabo la identificación correspondiente. En el ámbito forense, el método más usado "se basa en el análisis de la variabilidad de regiones cortas repetidas en tándem (en inglés: Short Tandem Repeats: STRs) de nuestro ADN nuclear, que ha adquirido un poder de discriminación enorme y también un alto grado de estandarización técnica a nivel mundial, lo que ha permitido un extenso intercambio de datos de $\mathrm{ADN}$ entre países y un control de calidad y acreditación en los laboratorios muy exigente" (cfr. "Recomendaciones para el uso correcto del análisis de ADN con finalidades forenses", Grupo de trabajo FIDE-Fundación Garrigues, 2019).

${ }^{26} \mathrm{Y}$ pese a ello, ¿es posible que algún día terminemos descubriendo que las técnicas actuales de identificación mediante el ADN son menos fiables de lo que ahora sabemos? Sí, por supuesto, en eso consiste también la ciencia; pero el derecho resuelve los casos con el conocimiento disponible en el momento. Y es la disponibilidad de ese conocimiento en el proceso judicial lo que debe ocupar a todos los participantes. 
La división del trabajo cognitivo en la prueba pericial: ...

tipo de pruebas periciales, el conocimiento experto es muy variado entre sí (lo que sabemos sobre la calidad de la prueba de ADN no es comparable con nuestro conocimiento sobre la validez y la calidad de otras pruebas forenses, por ejemplo), por lo que no puede ser encapsulado en uno o varios criterios delimitados; qué criterios serán relevantes dependerá del caso específico.

Ahora bien, lo que no se puede dejar de lado es que muchos de los criterios que se discuten nos brindan en el mejor de los casos información genérica sobre el conocimiento proposicional usado por el experto, sobre los métodos empleados o incluso sobre el experto mismo, pero aún falta información sobre la aplicación de dicho conocimiento y métodos al caso concreto, sobre lo que hizo el experto en el caso concreto. Para seguir con la prueba de ADN, pese a su validez y altísima fiabilidad en las mejores circunstancias, no todos los resultados de la prueba genética son iguales: ¿estaba degradada la muestra analizada?, ¿se respetó la cadena de custodia?, ¿se trata de transferencia secundaria o inocente?, y una larga lista de distintas preguntas que podrían afectar el valor probatorio de la prueba de ADN para el caso concreto. Por no hablar de que el ADN no nos dice nada sobre cómo una muestra llegó a la escena del delito, por lo que serán necesarias otras pruebas y, por supuesto, una buena interpretación del juez sobre lo que todas las pruebas dicen en su conjunto.

¿Tienen los jueces la información suficiente para responder al tipo de cuestiones anteriores o incluso para plantearse tales preguntas? Rimoldi, haciendo referencia a la etapa de la práctica de la prueba, nos dice:

.... el juez o la jueza debería estar atento a signos de irresponsabilidad epistémica, como la irracionalidad dialógica (tanto con él/ ella como con otros/as peritos/as); la arrogancia; y afirmaciones de certeza sobre las conclusiones y sobre los márgenes de error. En caso de encontrar estos signos, el juez o la jueza debería desconfiar fuertemente de la prueba pericial. Esto no significa que la prueba sea falsa, significa que el juez o la jueza no tiene las herramientas necesarias para valorar adecuadamente la prueba. No tiene la comprensión necesaria para hacerlo, porque la fuente de la que 
necesariamente debe depender presenta señales de vicios epistémicos que afectan la calidad de resultado ${ }^{27}$.

¿A qué herramientas se refiere Rimoldi? Supongo que se refiere al background que de hecho tiene el juez al acudir al juicio; sin embargo, diría que no podemos ceñirnos de manera exclusiva a él, sino en cómo ese complejo entramando cambia o qué parte del mismo se termina usando de hecho para tratar la prueba pericial del caso concreto. Lo que exigiría colocar al juzgador en una mejor situación cognoscitiva que le permita realizar una seria actividad epistémica a efectos de comprender el conocimiento experto que usará en su razonamiento probatorio. Esto, desde luego, requiere su actividad epistémica, pues no solo se trata de una mera transferencia de conocimientos de los expertos hacia el juez, sino que es menester su participación crítica. E insisto, la mejor etapa procesal para ello es la práctica de las pruebas, donde se puede obtener mayor información sobre la fiabilidad de las mismas y en la cual cobran relevancia las herramientas procesales que desarrollo en el artículo: preguntas de aclaración durante el contradictorio, asesores expertos para casos complejos, meta-periciales para complementar las pruebas periciales inicialmente presentadas y los careos entre expertos para afrontar los desacuerdos entre los mismos.

Herdy se pregunta: “¿cómo sería posible saber qué parte del conocimiento es suficiente si ni siquiera puedo entender el vocabulario técnico empleado, los supuestos fácticos de fondo o incluso lo que puede ser relevante?". La respuesta es que las herramientas procesales sugeridas ayudarían para que no se diera la situación de que el juez no pueda comprender el vocabulario técnico empleado, pero también para que sea más difícil que se dé el caso de que no entienda los supuestos fácticos de fondo. No niego que ambas situaciones pueden darse en la práctica, sobre todo en casos complejos, pero precisamente por ello hay que buscar las herramientas necesarias que ayuden a nuestros jueces, no quedarse de brazos cruzados

\footnotetext{
${ }^{27}$ Aquí hay otros tantos ejemplos que permitirían cuestionar la relevancia de la noción de irresponsabilidad epistémica. Por ejemplo, "las afirmaciones de certeza" que haga un perito en sí mismas nos deberían llevar a desconfiar de sus afirmaciones simple y sencillamente porque no puede haber certezas racionales; y ese presupuesto debería conformar el background de los jueces.
} 
La división del trabajo cognitivo en la prueba pericial: ...

solo señalando posibles problemas. Y cabe destacar que, si después del uso de las herramientas disponibles, el juez sigue sin comprender a los expertos para tomar una decisión sobre el caso, esta no depende necesariamente de esa fuente: puede haber más pruebas de otro tipo y hay ciertas reglas que permiten al juez decidir sin la prueba pericial.

Una vez más, qué contextos probatorios tendremos y qué herramientas procesales serán útiles dependerá de un complejo entramado entre el tipo de caso en juego, cuál es el derecho aplicable al mismo y qué han hecho o alegado las partes tanto en la admisión como en la práctica de la prueba pericial. En efecto, claramente el rol de las partes es fundamental en toda esa dinámica, dado que, en muchos de nuestros ordenamientos y prácticas actuales, en gran medida son ellas quienes decidirán la mayor parte de los cuestionamientos que se le hacen a los expertos que participan del proceso judicial, así como el rumbo de los argumentos y contraargumentos que a partir de ahí se enfatizarán. No podemos obviar esa dinámica y su impacto en la comprensión del juez: cuanto mejores sean los cuestionamientos de las partes, el debate entre ellas, mayor será la probabilidad de éxito en la comprensión de las afirmaciones de los expertos y su impacto en lo que cada una defiende. ${ }^{28}$

${ }^{28} \mathrm{Y}$, por supuesto, es parte de los costos para las partes. Al respecto, Herdy critica la afirmación de Allen sobre que el argumento del costo de educar al jurado no es especialmente relevante cuando en la inmensa mayoría de los litigios se hace esa ponderación en EUA. Por ello, Allen (2013, p. 52) afirma "the very question highlights in an important sense one of the bizarre aspects of the conventional discourse over expert testimony". Y a Herdy le parece bizarro que, a su vez, a Allen le parezca bizarro, dados ciertos desafíos político-económicos que ella señala. Sin embargo, creo que Herdy no está tomando en cuenta que el proceso judicial estadounidense depende en buena medida de los recursos de las partes y es a los costos para estas a las que se refiere Allen y no a los costos del sistema. Él vendría a decir algo así como que "las partes tienen que asumir el costo de educar al jurado para que decida", como asumen los costos de litigar.

Obviamente nada de lo anterior supone que los abogados tengan un objetivo desinteresado o imparcial, sabemos que están defendiendo a la parte que los contrató. Y como parte de su estrategia bien podría estar crear confusión, introducir más ruido, etc. De ahí la importancia también de asumir el contradictorio también como una herramienta cognoscitiva abierta a la participación del juez y de establecer ciertos límites a las partes. 


\section{El contextualismo en la prueba en el derecho}

¿Todo lo anterior exigiría, como afirma Rimoldi, que "el contexto en el que se da el intercambio epistémico entre jueces/zas y peritos/as deb[a] ser reconocido como un genuino contexto epistémico cuyas reglas y estándares de justificación no pueden extrapolarse del contexto científico"?

No entraré en el debate de la concepción epistemológica que subyace a lo anterior y que parece suponer que aquello que cuenta como justificación depende del contexto; ${ }^{29}$ en cambio, me concentraré en dos cuestiones jurídicas. En primer lugar, el esquema anterior, en el que se concibe la creación de una "comunidad epistémica de peritos y jueces", obvia a dos sujetos importantísimos en la discusión actual sobre la prueba pericial y a los que ya he hecho referencias anteriormente: las partes y la comunidad experta a la que pertenece el perito. Y, en segundo lugar, no permite vislumbrar el contextualismo que se da en la prueba en el derecho: es la suficiencia de la justificación, pero no la justificación en sí misma, la que depende del contexto. Vamos por partes.

Los sistemas jurídicos actuales han concedido a las partes un rol cada vez más importante en el control de la información que se presenta y se discute en un proceso judicial. Son ellas las que muchas veces no solo determinan el objeto del proceso sino también quienes ofrecen (al menos la mayoría de) las pruebas que luego debatirán en juicio oral. De hecho, bajo este esquema, en nuestros sistemas, donde tradicionalmente el único perito reconocido como tal era aquel designado por el juez, ahora ha entrado con fuerza el perito de parte cuya independencia está poco garantizada institucionalmente. Así pues, no solo es difícil pensar en una "comunidad" como la propuesta por Rimoldi, sino también en que además esté conformada únicamente por peritos y jueces.

Dado que las partes tienen sus propios intereses y que sus prácticas procesales para defenderlos no siempre coinciden con las mejores prácticas

29 Esto encajaría con la afirmación de Rimoldi: “[1]a justificación de teorías, creencias, hipótesis o proposiciones depende siempre del contexto particular o disciplinar en el que se estén considerando, y en este sentido una teoría, hipótesis, etc., puede estar justificada para un cierto contexto, pero no estarlo para otro". 
La división del trabajo cognitivo en la prueba pericial: ...

epistémicas, se deben establecer jurídicamente obligaciones concretas (no dejarlas a la[s] "práctica[s]") que debe cumplir todo perito hacia el tribunal y que sean conocidas por todos los participantes. Así, por ejemplo, que el perito esté obligado a ofrecer un informe pericial con argumentos completos y detallados considerando toda la información disponible sin importar a quién beneficie. Pero esa obligación, por supuesto, debe ir acompañada de la posibilidad de acceder a dicha información, de ahí la importancia de lo que en mi artículo inicial mencionaba respecto de mecanismos como el discovery. Tener un escenario normativo claro de qué corresponde hacer a cada uno favorecería además la seguridad jurídica de todos.

No es la idea de comunidad lo que mejor describiría los posibles intercambios argumentativos, aunque claramente encontramos un aspecto social en donde participan diversos sujetos con roles (e intereses) distintos. En ese ámbito debe preverse y ejercerse una división del trabajo cognitivo para optimizar los esfuerzos de todos a efectos de llegar sistemáticamente a decisiones judiciales justificadas a partir de las pruebas admitidas y practicadas. Ello no exige la constitución de una comunidad, sino un mínimo de cooperación de los participantes, que es incluso compatible con sus roles e intereses. Al abogado de una parte, sea directamente o mediante su perito, le conviene cuestionar al perito de la contraparte, por ejemplo, poniendo sobre la mesa sus sesgos cognitivos provenientes de la información brindada o incluso de su contacto con información sobre el caso o sus sesgos disposicionales o los implícitos que podrían derivarse de la selección misma. Esos cuestionamientos darán información al juez para valorar la prueba pericial y podrían también hacer ganar su caso al abogado.

En el mundo jurídico no se espera que los jueces y los expertos creen de alguna manera elementos sobre lo que cuenta como justificado para ambos; en el derecho se espera que el perito use lo que en su contexto profesional esté probado e informe sobre ello. Y ahí cobra sentido voltear a ver a las comunidades expertas que de muy diversas maneras no solo establecen ciertos patrones de conducta en el ejercicio profesional, sino que además tienen un conjunto de mecanismos para la validación del conocimiento y/o las prácticas que conforman sus áreas de conocimiento. Hay sistemas jurídicos que han aprendido sobre la importancia de acudir a las comunidades expertas para conocer los datos que se tienen sobre la validez y la fiabilidad 
de métodos, técnicas, etc., que son usados por peritos particulares; pero también hay sistemas jurídicos que han incentivado (con independencia de la prueba pericial de un caso concreto) que los grupos de expertos se preocupen por la aptitud de quienes practican su profesión. Ese tipo de actividades de los grupos de expertos redundan en la posibilidad de tener mejores peritos en el proceso judicial y el empleo de conocimiento fundado y, por ello, también juegan un importantísimo rol en la división de la tarea cognitiva correspondiente.

¿Lo anterior quiere decir que no hay espacio para el contextualismo? Por supuesto que lo hay: en la suficiencia de justificación, pero no en qué cuenta como justificado. El estándar de prueba es la regla que establece cuándo el conjunto de pruebas es suficiente para dar por probados los hechos del caso. La discusión sobre la suficiencia no se limita a un tipo de prueba, como la pericial, sino que el juez debe considerar conjuntamente todas las pruebas que han sido admitidas y practicadas. Ahora bien, cuán exigente debe ser el estándar nos lleva también a una discusión jurídica compleja, porque supone no solo decidir qué tipo de error es más costoso, sino que implica en algunos casos tomar en cuenta también las dificultades probatorias del tipo de hechos que se tienen que probar. Así pues, esta discusión no puede ser calificada como un "único contexto", al menos se tiene que distinguir el penal y el civil; y, aún más, dentro de ellos también podrían preverse estándares de prueba distintos para diferentes tipos de casos. ${ }^{30}$ Con todo, determinar el grado de suficiencia probatoria es una discusión político-moral que desafortunadamente seguimos sin ver reflejada en estándares de prueba en nuestros ordenamientos; pero esta situación no es un tema que se pueda resolver en el contexto de la prueba pericial, ${ }^{31}$ aunque por supuesto tiene implicaciones para ella. Por ejemplo, de manera muy breve: el estándar de prueba jurídico y el estándar de prueba científico no necesariamente deben coincidir dado que lo que hay en juego en cada uno de esos contextos puede ser muy distinto. Sin embargo, si no tenemos claro cuál es el estándar jurídico, será difícil o imposible un debate sobre

${ }^{30}$ Sobre los estándares de prueba en el derecho, véase Ferrer (2019).

${ }^{31}$ Mucho menos en una hipotética comunidad de jueces y peritos; es una decisión que correspondería al legislador. 
si los bienes en juego en el derecho exigen (o no) un estándar más alto o más bajo que el empleado por los expertos.

En definitiva, sea cual sea el estándar de prueba jurídico, cuánto más determinante sea la prueba pericial para su satisfacción, mayor nivel de comprensión tiene que tener el juez. Cuanta mayor comprensión judicial se requiera, más exigente debe ser la práctica de la prueba pericial, dado que es ahí donde se puede obtener y discutir la mayor información sobre la fiabilidad de las afirmaciones periciales. Para ello, ayudaría mucho tener buenos abogados cuestionando adecuadamente a los expertos, pero también es necesario prever herramientas para ayudar a la labor judicial en los distintos contextos probatorios que se enfrentan cuando hay conocimiento experto de por medio.

\section{Bibliografía}

Allen, R. (2013). The Conceptual Challenge of Expert Evidence. Revista Discusiones Filosóficas, 14(23), pp. 41-65.

Devis Echandía, H. (1972). Cientificidad de la prueba, en relación principalmente con los dictámenes periciales y la libertad de apreciación del juzgador. Revista de Derecho Procesal Iberoamericana, 1, 41-66.

Dror, I. (2018). Biases in forensic experts. Science, 360(6386), p. 243.

Ferrer, J. (2019). Prolegómenos para una teoría sobre los estándares de prueba. En Papayannis, D. y Pereira, E. (eds.), Filosofía del derecho privado. Madrid-Barcelona: Marcial Pons.

Freckelton, I., Goodman-Delahunty. J., Horan, J. y McKimmie, B. (2016). Expert Evidence and Criminal Jury Trials. Oxford: Oxford University Press. Grupo de trabajo FIDE-Fundación Garrigues (2019). Recomendaciones para el uso correcto del análisis de ADN con finalidades forenses.

Haack, S. (2020). Filosofía del derecho y de la prueba. Perspectivas pragmatistas. Madrid-Barcelona: Marcial Pons.

Lord Chief Justice, Lay Magistrates. Northern Ireland: Judiciary Ni. Recuperado de: https://judiciaryni.uk/lay-magistrates

Putnam, H. (1975). Mind, Language and Reality. Cambridge: Cambridge University Press. 
Kitcher, P. (1990). The division of cognitive labor. The Journal of Philosophy, 87(1), 5-22.

Salmon, W. (1984). Logic. New Jersey: Prentice Hill.

Vázquez, C. (2015). De la prueba científica a la prueba pericial. MadridBarcelona: Marcial Pons.

Walton, D. (1997). Appeal to Expert Opinion: Arguments from Authority. University Park: Pennsylvania State University Press.

Walton, D. (2008). Informal Logic. A pragmatic approach (2. $\left.{ }^{\mathrm{a}} \mathrm{ed}.\right)$. Cambridge: Cambridge University Press.

Woods, J. y Walton, D. (1974). Argumentum ad Verecundiam. Philosophy \& Rhetoric, 7(3), 135-153. 